\title{
Compliance and efficacy of extended release Metformin and immediate release thrice daily Metformin in type-2 Diabetes Mellitus- A comparative study
}

\author{
${ }^{1}$ Dr. Jitendra kumar, ${ }^{2}$ Dr.Sunil Kumar, ${ }^{3}$ Dr. Vandana, ${ }^{4}$ Dr Prof. S. Nag, \\ ${ }^{5}$ Dr. Amit Kumar, \\ ${ }^{I}$ Asst. Prof. Dept.of Medicine, Narayan Med. College \&hospital. Jamuhar, Sasaram, Rohtas, Bihar \\ ${ }^{2}$ Asst. Prof. Dept. of Community Medicin, Narayan Med. College. \\ ${ }^{3}$ Asst. Prof. Dept. of Physiology, Narayan Med.Coll. \\ ${ }^{4}$ Prof. \&HOD Anesthesiology, Narayan Med. Coll. \\ ${ }^{5}$ Asst. Prof. Dept. of Pharmacology, Narayan Med. Coll.
}

\begin{abstract}
:
Aim of study: Metformin is a first-line pharmacological treatment for patients with type 2 diabetes mellitus because of its favorable overall profile, including its glucose-lowering ability, weight-neutral effects, and low risk of hypoglycemia; however, gastrointestinal (GI) intolerance may limit use in some patients. Extendedrelease metformin improves GI tolerability, allows once-daily dosing,improves compliance in patients.
\end{abstract}

Conclusion: Patients with type 2 DM who had been receiving thrice daily MIR achieved comparable glycemic control when therapy was switched to once or twice daily MXR at the same daily dose.

\section{Introduction}

Metformin hydrochloride has been widely used as an effective and generally well-tolerated glucoselowering agent for $>40$ years and is the most frequently prescribed first-line therapy for patients with type 2 diabetes . Metformin typically reduces basal and postprandial hyperglycemia by $\sim 25 \%$ in $>90 \%$ of patients when given alone or with other therapies during a program of managed care. An extended release formulation (MXR approved in October 2000 allows once daily dosing. This newer formulation release the active drug through active hydrated polymers which expand after uptake of fluids. This prolongs gastric residence time. Extended-release metformin showed slightly lower maximum concentrations, longer times to maximum concentration ( $7-8$ vs. $4-5 \mathrm{~h}$ ), and similar bioavailability compared with immediate-release products. This extended release could potentially reduce dosing frequency to once daily compared with two or three times daily for immediate-release formulations. which produces slower drug action in the upper GI tract allowing once a day dosing.

\section{Materials And Methods}

We conducted a clinical trial involving 75 patients with T2DM who were on at least $1 \mathrm{gm}$ of MIR with or without other antihyperglycemic agents. Eligibility criteria included an age of atleast 40 years, a BMI of 20 or higher, and fasting capillary glucose of $<120 \mathrm{mg} \%$

Patient were required to be free of symptomatic of T2DM,significant renal dysfunction( serum creatinine $1.5 \mathrm{mg} \%$ for men,> $1.4 \mathrm{mg} \%$ for women) hepatic dysfunction, congestive heart failure, major psychiatric disorder ,alcohol and/ or substance abuse ,seizure disorders, or a history of malignancy or steroid intake, pregnant or breastfeeding women were excluded from the study. Patients could be receiving long term insulin therapy or any other anti hyperglycemic therapy apart from MIR. After a lead in period of three months, during which patients were on weight maintenance diet and 1000-2000 mg of MIR (in 2 or 3 divided doses) all patients who were at least $80 \%$ compliant with the drug treatment(as determined by tablets count and interviews) were entered in the study.

At the visit of trial(visit 0 ) all eligible patients with T2DM were switched over to one particular brand of MIR while the dose of MIR and other hypoglycemic agents were kept the same. Baseline measurement included a two point glucose profile (Fasting, 2 hour PP), Serum Lipid profile (Total Cholesterol), low density lipoprotein cholesterol (LDL), High Density Lipoprotein (HDL) and Triglycerides (TG) are concentrations, standard laboratory tests (haematology, serum chemistry profile, urine analysis) and electrocardiography (ECG). A record of body weight, blood pressure and other vital signs were maintained. After one month (Visit 1) a subjects were assessed and then switched over to 500mg lesser dose of MXR which was given in the form of a single dose after dinner. Whereas the other antihyperglycemic agents were continued. Patients were subsequently called for 3 or more monthly intervals. 
During reassessment, at each visit patients are particularly asked about any possible side effects in addition to change in dosage schedule as per protocol. At each visit two point blood glucose estimation was done. Ten patients were excluded from the study which included 7 patients who did not follow till the end of the study, 2 who had poor compliance, 1 who discontinued MXR because of the skin rash. The data from the remaining 40 subjects were analysed for the results. The baseline characteristic of 10 excluded patients were not significantly different from those of study subjects.

Capillary blood glucose was measured with glucose monitor. Standardization of equipments was frequently done with the help of quality controls of known concentration provided with the instruments. Fasting serum triglycerides, total cholesterol and HDL cholesterol were measured and low density cholesterol was calculated. All fasting estimation were conducted after a minimum fast of 8 hours. Compliance was assessed by reviewing the tablets count conducted at each study visit. Additionally patients were asked to confirm their compliance with therapy of each visit. Acceptance compliance was defined as $>80 \%$ of expected study drug consumption. Efficacy of the drug was assessed by comparing the mean change in two point glucose profile at each visit. $\mathrm{P}$ value of $<0.05$ was taken as statistically significant.

\section{Results}

The demographic and baseline clinical characteristics of the study subjects are summarized in Table1. Age of the patients ranged from 40 to 74 years with $12(30 \%)$ patients having age of 65 years or more. Duration of diabetes ranged from 3 months to 22 years with $25(56 \%)$ having diabetes of 10 years duration or more. Majority of patients were obese; 22(55\%) had BMI of 25 or more. History of HTN was observed in 31(77.5\%) with $45 \%$ having a BP of $140 / 90 \mathrm{mmhg}$ or more. Of all 40 patients $33(82.5 \%)$ were on other anti-hyperglycemic medication which included Insulin in $10(25 \%)$ patients and sulfonylureas in 23(57.5\%). Of 10 patients on Insulin 2 were also on $15 \mathrm{mg}$ per day of pioglitazone in addition. Sulfonylurease used were Glipizide (3 patients), Glimpiride(9 patients) and Gliclazide(11 patients). Initial assessment revealed peripheral neuropathy in $30(75 \%)$, retinopathy in $16(40 \%)$, nephropathy in $10(25 \%)$, coronary artery disease in $4(10 \%)$ and previous stroke in $2(5 \%)$.

Of 16 patients with retinopathy 1 had proliferative retinopathy. 9 patients had proteinuria of more than $300 \mathrm{mg} / 24$ hours. Fasting and post breakfast, glucose profile study of the subjects was done at visit 1,2,3 and 4 . Fasting glucose was less than $120 \mathrm{mg} / \mathrm{dl}$ in $80 \%, 63 \%, 73 \%$ and $70 \%$ and mean post prandial glucose was $<180 \mathrm{mg} / \mathrm{dl}$ in $90 \%, 75 \%, 73 \%$ and $90 \%$ at visits $1,2,3$ and 4 respectively. Thought these differences were not statisctically significant. Table 2 shows comparative blood glucose levels with different Metformin regimes used in one daily sequence. As is evident form the table mean level of both fasting as well as post prandial glucose levels increase significantly after patients were switched to MXR when the dose was 500mg lesser than prior used dose of MIR. However glucose profile was again comparable to baseline (except Post Prandial Glucose) when dose of MXR was increased back to original dose of MIR after one month. At visit 4 when patients were on 2 split doses of MXR for one month, mean fasting glucose was $118 \pm 18 \mathrm{mg} / \mathrm{dl}$ compared to baseline value of $109 \pm 20(\mathrm{p}=0.029)$ but all post prandial values were comparable to baseline values of visit 1 . Mean weight at the end of 3 months of MXR was $68.7 \pm 10.2$ as compared to $69.6 \pm 10.8 \mathrm{~kg}$ at baseline $(\mathrm{p}=0.020)$. Lipid profile after 3 months of MXR including mean TC (192 $\pm 36 \mathrm{mg} / \mathrm{dl})$, LDL (124 $\pm 20 \mathrm{mg} / \mathrm{dl})$, HDL $(41 \pm 5 \mathrm{mg} / \mathrm{dl})$ and TG $(132 \pm 35 \mathrm{mg} / \mathrm{dl})$ was not significantly different from that of baseline. There were not many new side effects with any regiments of MXR, 2 patients complained of diarrhea and one had loss of appetite and flatulence that settled spontaneously. Only one patient required reduction in Insulin doses due to minor hypoglycemic episodes. No patients in the trial required medical assistance to manage hypoglycemia.

TABLE 1 : Baseline characteristics of the study population

\begin{tabular}{|l|l|}
\hline Characteristic $(\mathrm{mm})$ & Value \\
\hline Mean Age (years) & $51.2 \pm 8.4$ \\
\hline Mean duration of diabetes & $12.4 \pm 5.6$ \\
\hline Mean BMI (Kg/m2) & $24.8 \pm 3.7$ \\
\hline Mean systolic BP $(\mathrm{mmhg})$ & $134.5 \pm 16.6$ \\
\hline Mean diastolic BP(mmhg) & $78.6 \pm 8.2$ \\
\hline Mean fasting glucose (mg/dl) & $112.25 \pm 21$ \\
\hline Mean Post Prandial glucose (mg/dl) & $155.5 \pm 39.2$ \\
\hline Mean Creatinine $(\mathrm{mg} / \mathrm{dl})$ & $0.9 \pm 0.3$ \\
\hline Lipid Profile & \\
\hline Mean TC & $192 \pm 36$ \\
\hline Mean LDL & $124 \pm 20$ \\
\hline Mean HDL & $41 \pm 5$ \\
\hline Mean TG & $132 \pm 35$ \\
\hline & \\
\hline
\end{tabular}




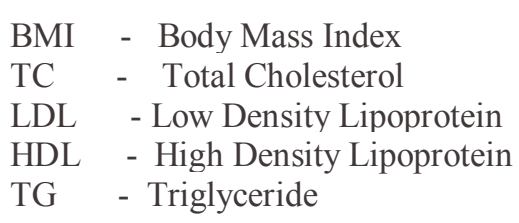

TABLE 2: Mean fasting and postprandial glucose levels with different regimes of metformin prescriped for one month.

\begin{tabular}{|l|l|l|}
\hline & Mean fasting glucose $(\mathrm{mg} / \mathrm{dl})$ & Mean post prandial $(\mathrm{mg} / \mathrm{dl})$ \\
\hline Visit 1 & $109 \pm 20$ & $154 \pm 35$ \\
\hline Visit 2 & $112 \pm 23$ & $152 \pm 42$ \\
\hline Visit 3 & $110 \pm 21$ & $156 \pm 37$ \\
\hline Visit 4 & $118 \pm 18$ & $160 \pm 43$ \\
\hline
\end{tabular}

Visits were monthly after taking MIR in a dose of 1-2 gm/day (Visit 1 ); Mxr with dose 0.5 gm less than baseline dose of MIR (Visit 2) ; MXR 1-2 gm/day with dose same as baseline MIR (Visit3), and MXR 1$2 \mathrm{gm} /$ day in divided doses with dose same as baselineMIR (Visit 4).

\section{Discussion}

We conducted this study to determine the efficacy and tolerability of extended release Metformin (MXR) in patients with T2DM who had achieved moderate or good glycemic control with immediate release Metformin (MIR) alone or in combination with other antihyperglycemic agents. In this small group of patients with T2DM MXR and MIR had nearly identical efficacy in reducing plasma glucose concentrations. Our data suggests that MXR is more convenient and well tolerated with insignificant rates of adverse reactions.

Metformin is a Biguanide that has been used worldwide for the treatment of T2DM for the last four decades. It has been used increasingly as a first line agent for T2DM, both as monotherapy and as a component of combination regiments. The ADA/EASD guidelines mention its use in patients irrespective of age, body weight and degree of baseline hyperglycaemia. Its not therefore surprising that in populations as diverse as this that the use of standard immediate release metformin is problematic in some patient groups leading to difficulties with dose optimization. It improves glycemic control by enhancing insulin sensitivity in liver and muscles, It is not associated with hypoglycemia. Improved metabolic control with Metformin does not cause weight gain and may lead to weight loss. Metformin also has beneficial effects on several cardiovascular risk factor such as dyslipidemia, elevated plasma plasminogen activated inhibitor, other fibrinlolytic abnormalities and hyperinsulinemia and insulin resistance. Metformin is not metabolized by the liver and excreted intact in urine. Elimination is characterized by a rapid initial phase during which $90 \%$ appears in the urin within 8-12 hour and a slower elimination phase with a half life of 12-20 hour. Metformin is generally given in divided doses 2-3 times a day. The usual starting dose is $500 \mathrm{mg}$ twice daily. The dose is increased or decreased 250 $500 \mathrm{mg}$ /day every two weeks untils the desired level of glycemic control is achieved or a maximum dose of 2000 $\mathrm{mg}$ /day is reached. Until recently the conventional MIR formulation administered twice or thrice daily has been used as first line therapy but this formulation maybe less than optimal in terms of patients compliant with daily therapy.

Metformin-XR was developed with the intention of enabling once-daily dosing of metformin, and of improving its gastrointestinal tolerability. This extended release formulation of metformin uses a patented proprietary GelShield Diffusion System technology which provides a once-daily dosing option for patients with type 2 diabetes. When administered with the evening meal, the GelShield Diffusion System of metformin-XR releases metformin over the dosing period and works in conjunction with the normal physiology of slowed nocturnal and postprandial GI emptying, resulting in a once-daily dosing option.

Taking into consideration its importance in therapeutics and its pharmacodynamics advantages over other agents. The sustained released preparations of Metformin not only should have improved patients compliance but also might improve the pathophysiology of the disease. The extended release metformin tablet releases metformin from the upper GI tract for a longer period of time thereby delaying its entry into the systemic circulation. Metered release of metformin from the tablet and administration with the evening meal when gastric residence time is at its longest are thought to be factors in explaining better tolerability.

Our study was designed to assess glycemic control after a switch from MIR to MXR. The design of the study was also to determine tolerability a single dose of MXR given at bedtime vs 2 divided doses of MXR. Patients enrolled in the study had established T2DM while receiving 1000-2000 mg/day BID or TID for 12 weeks and mean fasting glucose of $<120 \mathrm{mg} / \mathrm{dl}$. Patients when switched to MXR 1000 or $1500 \mathrm{mg}$ once daily achieved indices of glycemic control that were comparable to those of patients who continued MIR therapy. 


\section{Conclusion}

Switchover to metformin XR resulted in a significant improvement in gastrointestinal tolerability with marked reductions in diarrhoea and nausea. Improved patient satisfaction and well being led to the XR once-aday tablet being preferred in a high majority of patients. When considered alongside the comparative efficacy of the metformin XR tablet versus standard metformin, this new formulation of metformin has the potential to improve compliance and long-term health outcomes. This is the goal if the aim of therapy is to reduce excess morbidity and mortality in patients with type 2 diabetes.

\section{References}

[1]. Marathe P, Turner K. Steady-state pharmacokinetics of the metformin extended-release tablet versus immediate-release metformin in healthy subjects Diabetes 2002; 51 (suppl 2):A474

[2]. Schwartz S, Fonseca V, Berner B, Cramer M, Chiang YK, et al. (2006) Efficacy, tolerability and safety of novel once-daily XR metformin in patients with type 2 diabetes. Diabetes Care 29: 759-763.

[3]. Lawrence Blonde, George E. Dailey, Serge A. Jabbour, Charles A. Reasner, Donna J. Mills Curr Med Res Opin. 2004;20(4)

[4]. Gastrointestinal tolerability of extended-release metformin tablets compared to immediate-release metformin tablets: results of a retrospective cohort study Lawrence Blonde, George E. Dailey, Serge A. Jabbour, Charles A. Reasner, Donna J. Mills (doi: 10.1185/030079904125003278)

[5]. Donnelly LA, Morris AD, Pearson ER: Adherence in patients transferred from immediate release metformin to a sustained release formulation: a population-based study._Diabetes Obesity and Metabolism 2009, 11:338-342 